\title{
Flow field analyses of a porous membrane-separated, double-layered microfluidic chip for cell co-culture
}

\author{
Shenbao Chen ${ }^{1,2}$. Jian Xue $\mathrm{Xu}^{2,3}$. Jinrong Hu ${ }^{1,2}$. Qihan Ding ${ }^{1,2} \cdot$ Lüwen Zhou $^{1,4}$. Shiliang Feng ${ }^{1,4}$. Yuhong Cui ${ }^{5}$. \\ Shouqin Lü ${ }^{1,2} \cdot$ Mian Long ${ }^{1,2}$
}

Received: 7 January 2020 / Revised: 20 February 2020 / Accepted: 11 March 2020 / Published online: 9 May 2020

(C) The Chinese Society of Theoretical and Applied Mechanics and Springer-Verlag GmbH Germany, part of Springer Nature 2020

\begin{abstract}
Organs-on-chips composed of a porous membrane-separated, double-layered channels are used widely in elucidating the effects of cell co-culture and flow shear on biological functions. While the diversity of channel geometry and membrane permeability is applied, their quantitative correlation with flow features is still unclear. Immersed boundary methods (IBM) simulations and theoretical modelling were performed in this study. Numerical simulations showed that channel length, height and membrane permeability jointly regulated the flow features of flux, penetration velocity and wall shear stress (WSS). Increase of channel length, lower channel height or membrane permeability monotonically reduced the flow flux, velocity and WSS in upper channel before reaching a plateau. While the flow flux in lower channel monotonically increased with the increase of each factor, the WSS surprisingly exhibited a biphasic pattern with first increase and then decrease with increase of lower channel height. Moreover, the transition threshold of maximum WSS was sensitive to the channel length and membrane permeability. Theoretical modeling, integrating the transmembrane pressure difference and inlet flow flux with chip geometry and membrane permeability, was in good agreement with IBM simulations. These analyses provided theoretical bases for optimizing flow-specified chip design and evaluating flow microenvironments of in vivo tissue.
\end{abstract}

Keywords Immersed boundary method $\cdot$ Porous membrane $\cdot$ Wall shear stress $\cdot$ Penetration velocity $\cdot$ Microfluidic chip

\section{Introduction}

Electronic supplementary material The online version of this article (https://doi.org/10.1007/s10409-020-00953-4) contains supplementary material, which is available to authorized users.

Shouqin Lü

lsq@imech.ac.cn

$\bowtie$ Mian Long

mlong@imech.ac.cn

1 Center of Biomechanics and Bioengineering, Key Laboratory of Microgravity (National Microgravity Laboratory), Beijing Key Laboratory of Engineered Construction and

Mechanobiology, and CAS Center for Excellence in Complex System Mechanics, Institute of Mechanics, Chinese Academy of Sciences, Beijing 100190, China

2 School of Engineering Science, University of Chinese Academy of Sciences, Beijing 100049, China

3 State Key Laboratory of Nonlinear Mechanics (LNM), Institute of Mechanics, Chinese Academy of Sciences, Beijing 100190, China

4 Faculty of Mechanical Engineering and Mechanics, Ningbo University, Ningbo 315211, Zhejiang, China
Coexistence and arrangement of multiple cell types with defined spatial locations as well as physical and mechanical microenvironments within specific tissues or organs are crucial for their corresponding functions. With developments of microfabricating and microfluidic technologies, in vitro organs-on-chips such as liver [1], kidney [2], lung [3] and intestine [4] have become popular platforms for investigating the organ-specific biological mechanisms, disease progresses and therapeutic treatments [5]. Various types of organs-onchips have been developed in different ways. The simplest way is to randomly mix different types of cells in a co-culture system. For example, co-culture of adult rat hepatocytes directly with another liver epithelial cell type helps the maintenance of albumin secretion of hepatocytes [6]. While this type of liver chip provides effective cell-cell contact and communication, the configuration of spatial localization among

5 Department of Mechanics, Tianjin University, Tianjin 300072, China 
different cell types is precluded. On the other hand, micropatterned co-culture can precisely define the spatial location and arrangement of various cells but cannot separately control the fluid flow over different cell types [7]. Co-culture chips reconstructed within three-dimensional scaffolds can also better mimic the spatial configuration among different types of cells with enlarged contact surfaces, but it is still limited in simulating the physiological microenvironments of blood flow and nutrient supply [8].

Fluid flow is crucial in many biological processes as well as in reconstructing functional organs-on-chips. In liver, blood perfusion has been demonstrated to be key for liver growth [9]. Shear stress promotes vasculature growth in embryonic cardiovascular development [10]. Fluid flow accelerates the phenotypic changes from corneal fibroblasts or NIH 3T3 cells to myofibroblasts [11] and alters gene expression and biosynthesis of endothelial cells [12]. Meanwhile, several microfluidic platforms using micropillar [13] or microtunnel arrays [14] or porous membranes [15] as endothelial-like barriers are developed to mimic the permeability of vascular endothelium. These platforms have the potentials to simultaneously coordinate the communications and spatial localization among different cell types with the fluid dynamics microenvironment of those culturing cells. In addition, the cells can be easily cultured on the two opposite sides of a porous membrane and exposed to shear stress from both sides $[1,3,16,17]$. It is also possible to receive unique inputs or perturbations on their apical and basolateral surfaces when the cells are seeded on one side of a permeable membrane. Thus, this type of a porous membrane-separated, double-layered microfluidic chip is considered as an effective model for those organs with abundant blood supply and highly permeable endothelium.

Specifically, in liver, at least four types of hepatic cells (hepatocytes, hepatic stellate cells, liver sinusoidal endothelial cells (LSECs), and Kupffer cells) together constitute the unique liver sinusoids, in which the sinusoidal channel and Disse space are separated by a porous vascular bed consisting of a layer of LSECs. Fenestrae on LSECs allow blood to penetrate through endothelium and access to parenchymal cells. To understand the sinusoidal functions under physiologically-like cell location configuration and fenestrated features of sinusoidal endothelium, a porous membrane-separated, double-layered microfluidic chip has been developed in our lab [1]. Our results show that the hydrodynamic features in the double-layer liver chip is important in the enhancement of liver-specific functions [1]. Similar results are also obtained in other works to demonstrate the importance of hydrodynamic design for liver chips [18, 19]. While the effectiveness of porous membrane-separated channels or similar devices are validated in widely varied channel geometries and membrane permeabilities, the impacts of mechanical microenvironments on biological functions are still open mainly due to the lack of criteria for this type of chip design and evaluation of flow features.

Porous membranes widely used as tissue barrier or in co-culture models have a wide range of permeability [15], wherein pore size ranging from $3 \mathrm{~nm}$ to $10 \mu \mathrm{m}$, membrane thickness from $15 \mathrm{~nm}$ to $50 \mu \mathrm{m}$, and the resulted hydraulic permeability from $10^{-9}$ to $10^{-5} \mathrm{~Pa} \cdot \mathrm{s} \cdot \mathrm{m}^{-3}$. Channel sizes also vary significantly, as exemplified in liver sinusoidal chips with the channel length from about 8 to $34 \mathrm{~mm}$ and the upper and lower channel heights from tens to hundreds of micrometers [18-20]. These diversities of chip geometry and porous membrane permeability result in the variety of flow features in the channels and further affect the biological functions of in vitro organ-on-chips. To optimize the microfluidic design of this type of porous membrane-separated organs-on-chips, it is required to quantify the fluid characteristics inside the chip and determine the rules of membrane porosity selection and chip geometry design. Here the dependences of flow features on chip geometry and membrane permeability were systematically evaluated using the IBM numerical simulations in a porous membrane-separated, double-layered microfluidic chip channel. Corresponding theoretical models were developed to correlate the hydrodynamic characteristics with chip geometry and membrane permeability. Our results offered theoretical bases for evaluating the mechanical microenvironment of membrane-separated organs-on-chips.

\section{Methods}

\subsection{Chip set-up}

Numerical simulation and theoretical prediction were based on a three-dimensional microfluidic chip with two parallel rectangular-cross-section channels separated by a porous membrane for mimicking liver sinusoid structure [1] (Fig. 1a). The inlet and outlet of the chip were set at the left and right ends of the upper channel, respectively. Since the channel width is at least one order-of-magnitude larger than the heights of both upper and lower channels and the chip configuration is symmetric, the chip geometry was thus simplified to a two-dimensional (2D) model in our numerical simulation (Fig. 1b).

\subsection{Simulation method}

IBM was used to construct the simulation framework in this work. Simulations were run on the MATLAB platform, and the corresponding codes were modified from an open-source package developed by Battista et al. [21] and distributed on the website http://www.github.com/nickabattista/IB2d. 2D Eulerian grid and one-dimensional Lagrangian gird were used for representing fluid domain and immersed bound- 
aries, respectively. Channel walls and the porous membrane were both treated as immersed boundaries using rigid nodes (Fig. 1b). In addition, the porous membrane was modeled based on Darcy's Law and Peskin's method [22]. Here the fluid velocity through a porous boundary is proportional to the pressure gradient between the two sides of this boundary (Fig. 1d) and given by,

$U_{\mathrm{p}}=-\frac{\kappa[p]}{\mu h}=-\frac{\kappa}{\mu h} \cdot \frac{\boldsymbol{F} \cdot \boldsymbol{n}}{\left\|X_{s}\right\|}$,

where $U_{\mathrm{p}}$ is the normal slip velocity through the porous membrane, $\mu$ is the fluid viscosity, $\kappa$ is the membrane permeability, $h$ is the membrane's thickness, $[p]$ is the pressure gradient across the boundary, $\boldsymbol{F}$ is the constrain force density on membrane nodes, $\mathbf{n}$ is a unit vector normal to the membrane, and $\boldsymbol{X}_{s}$ is the differentiation of the membrane coordinates $\boldsymbol{X}(s, t)$ in curvilinear systems as $\partial \boldsymbol{X}(s, t) / \partial s$. Two governing equations of the momentum conservation equation (Eq. (2)) and continuity equation (Eq. (3)) are expressed as the following:

$$
\begin{aligned}
& \rho\left(\frac{\partial u(\boldsymbol{x}, t)}{\partial t}+\boldsymbol{u}(\boldsymbol{x}, t) \cdot \nabla \boldsymbol{u}(\boldsymbol{x}, t)\right) \\
& \quad=-\nabla p(\boldsymbol{x}, t)+\mu \Delta \boldsymbol{u}(\boldsymbol{x}, t)+\boldsymbol{f}(\boldsymbol{x}, t),
\end{aligned}
$$

$\nabla \cdot \boldsymbol{u}(\boldsymbol{x}, t)=0$

Here $\boldsymbol{u}(\boldsymbol{x}, t)$ is the fluid velocity, $p(\boldsymbol{x}, t)$ is the pressure, and $f(\boldsymbol{x}, t)$ is the force density (force per unit area) applied to the fluid by immersed boundaries. The independent variables are the position $\boldsymbol{x}=(x, y)$ and the time $t$. Numerical calculations were performed and iterated on the followed four steps. Firstly, the constrain force density $\boldsymbol{F}(s, t)$ on an immersed boundary node is calculated upon the distance between its current position $p$ and its balance position $p_{0}$ (Fig. $1 \mathrm{c}_{\mathrm{i}}$ ). Then, $\boldsymbol{F}$ is dispersed to the neighboring fluid region using a Delta Kernel function (Eq. (4)) (Fig. 1 $\mathrm{c}_{\mathrm{ii}}$ ). Next, the fluid velocity $\boldsymbol{u}$ is updated by solving the governing equation (Eqs. (2), (3)) (Fig. $1 c_{i i i}$ ). Lastly, the node velocity $\boldsymbol{U}$ is updated by interpolating $\boldsymbol{u}$ using a Delta Kernel function (Eq. (5)) (Fig. 1 $\mathrm{c}_{\mathrm{iv}}$ ). Regularized Delta Kernel function in the cosine form was used for implementing force distribution and velocity interpolation (Eqs. (6), (7)):

$$
\begin{aligned}
& \boldsymbol{f}(\boldsymbol{x}, t)=\int \boldsymbol{F}(s, t) \delta(\boldsymbol{x}-\boldsymbol{X}(s, t)) \mathrm{d} s, \\
& \boldsymbol{U}(\boldsymbol{X}(s, t), t)=-U_{\mathrm{p}} \boldsymbol{n}+\int \boldsymbol{u}(\boldsymbol{x}, t) \delta(\boldsymbol{x}-\boldsymbol{X}(s, t)) \mathrm{d} \boldsymbol{x}, \\
& \Phi(r)= \begin{cases}\frac{1}{4}\left(1+\cos \left(\frac{\pi r}{2}\right)\right), & |r| \leq 2, \\
0, & |r|>2,\end{cases} \\
& \delta_{h}(\boldsymbol{x})=\frac{1}{h^{2}} \Phi\left(\frac{x}{h}\right) \Phi\left(\frac{y}{h}\right) .
\end{aligned}
$$

In the simulations, periodic boundary conditions were set along both $x$ - and $y$-directions and the fast Fourier transform (FFT) method was employed to solve the governing equations.

\subsection{Numerical simulation}

The simulation zone of the fluid was discretized into $2 \mathrm{D}$ constructed grids with grid size of $\mathrm{d} x=\mathrm{d} y=1 \mu \mathrm{m}$, and the immersed boundaries were dispersed into $1 \mathrm{D}$ constructed grids with half of the size of fluid grid, i.e., $\mathrm{d} s=0.5 \mu \mathrm{m}$. Time step was set to be one microsecond to make sure the Courant Number is low enough during the simulation $(<0.01)$. Finite difference approximations were used to discretize the Navier-Stokes equations on the Eulerian (fluid) grid as described in the literature [21]. The inlet zone was set on the left side of upper channel (red rectangle region) and the outlet zone was on the right side (Fig. 1b). External force density with the magnitude of $f=k_{\text {drive }} \cdot\left(u_{\text {target }}-u_{\text {current }}\right)$ was applied to the two meshwidth-wide fluid region at the inlet zone to drive the fluid at every timestep for target parabolic distributions, where $u_{\text {target }}$ was target velocity of the driven zone and $u_{\text {current }}$ was corresponding velocity at current time $t$. At the beginning of simulation $(t=0)$, the velocity was set as zero over entire flow field. The fluid at the inlet was then accelerated at $t>0$ and drove the downstream fluid and flow field inside the entire channel gradually became stable with the progress of simulation. All the parameters used were summarized in Table 1. Periodical boundary conditions were used for the fluid zone, and Newtonian flow was considered in this study. The Reynold's number in this study ranged between $\sim 0.01$ and $\sim 0.2$.

To validate the reliability of IBM simulations, flow field analyses for the same chip were also performed using FLUENT 6.3.26 with the default precision of 2 ddp, as described in our previous work [1]. The 2D computational fluid dynamics (CFD) model was built and meshed $(1 \mu \mathrm{m} \times 1 \mu \mathrm{m})$ by GAMBIT 2.0. The porous membrane was set as fluid region of $10 \mu \mathrm{m}$-thick using the porous zone model, with the viscous resistance set to be $1 \times 10^{20} \mathrm{~m}^{-2}$ in $x$-direction (main flow direction) and $2 \times 10^{12}, 1 \times 10^{14}, 3.33 \times 10^{14}$ or $3.33 \times$ $10^{15} \mathrm{~m}^{-2}$ in $y$-direction. Other boundaries were treated as rigid walls. The velocity distribution of the inlet was set as parabolic by a User Defined Function (UDF) file and the outlet condition was set as outflow.

\subsection{Data analysis in numerical calculations}

Chip flow features analyzed in this study included the flow flux of inlet, transmembrane pressure difference and $y$ direction penetration velocity along the porous membrane, $x$-direction velocity and wall shear stress (WSS) of both upper and lower channels. Wall shear stresses were calcu- 
Table 1 Parameter setting in numerical simulations

\begin{tabular}{llll}
\hline Parameters & Symbol & Value & Unit \\
\hline Fluid density & $\rho$ & 1000 & $\mathrm{~kg} / \mathrm{m}^{-3}$ \\
$\begin{array}{l}\text { Dynamic fluid } \\
\quad \text { viscosity }\end{array}$ & $\mu$ & $1,3,4,5,6$ & $\mathrm{cP}$ \\
Time step & $\mathrm{d} t$ & 1 & $\mu \mathrm{s}$ \\
Simulation time & $t$ & $0.01-0.1$ & $\mathrm{~s}$ \\
Lagrangian grid size & $\mathrm{d} s$ & 0.5 & $\mu \mathrm{m}$ \\
Eulerian grid size & $\mathrm{d} x$, dy & 1 & $\mu \mathrm{m}$ \\
$\begin{array}{l}\text { Channel length } \\
\text { Upper channel height }\end{array}$ & $L$ & $0.8-13$ & $\mathrm{~mm}$ \\
$\begin{array}{l}\text { Lower channel height } \\
\text { Membrane thickness }\end{array}$ & $H$ & $0.05,0.1$ & $\mathrm{~mm}$ \\
$\begin{array}{l}\text { Membrane } \\
\text { permeability }\end{array}$ & $\kappa$ & $0.006-0.18$ & $\mathrm{~mm}$ \\
$\begin{array}{l}\text { Stiffness coefficient of } \\
\text { target point spring }\end{array}$ & $k_{\text {target }}$ & $1 \times 10^{-4}$ & $\mathrm{~mm}$ \\
$\begin{array}{l}\text { Stiffness coefficient of } \\
\text { springs for driving }\end{array}$ & $k_{\text {drive }}$ & $2 \times 10^{9}$ & $\mathrm{~m}{ }^{2}$ \\
$\quad$ inlet fluid & & & $\mathrm{N} / \mathrm{m}^{3}$ \\
$\begin{array}{c}\text { Target maximum inlet } \\
\text { velocity }\end{array}$ & $U_{0}$ & $0.5,1.5,5.0$ & $\mathrm{~N} \cdot \mathrm{s} / \mathrm{m}^{4}$ \\
\hline
\end{tabular}

lated according to the steady transverse velocity profile of the vertical middle sections, based on the equation of $\tau=$ $4 \mu U_{\mathrm{m}} / H$, where $U_{\mathrm{m}}$ is the peak value of the parabolic velocity profile, $H$ is the channel height and $\mu$ is fluid viscosity. The impacts of chip length and height, porous membrane permeability, inlet velocity, and fluid viscosity on equilibrium state of flow field were systematically analyzed, where the flow equilibrium states were confirmed by tracking the average transmembrane pressure difference, the fluid penetration velocity and the velocity on vertical middle cross-section of lower channel (Fig. S1). Since the actual inlet velocities varied among different cases in the simulations (refer below), the calculated results including flow flux, flow velocity and wall shear stress were unified by multiplying a common factor of $1.67 / U_{\text {in }} \mathrm{mm} / \mathrm{s}$ for comparisons.

\subsection{Theoretical modelling}

In order to quantify the relationship between chip geometry, porous membrane permeability and flow features, a theoretical model was also established for an analogical porous membrane-separated, double-layered microfluidic chip upon the above numerical results and two basic assumptions. The outline and detailed derivation of the theoretical model were presented in both Sect. 3 and Supplemental document 1.

\section{Results}

\subsection{Basic flow features of porous membrane-separated, double-layered microfluidic chip}

We first tested the robustness of numerical simulations of chip flow. The time required to reach an equilibration state was evaluated by tracking flow evolution. All the flow parameters reached plateaus within several microseconds (see Fig. S1 in Supplemental document 1), including average transmembrane pressure difference (Fig. S1a) and corresponding penetration velocity (Fig. S1b) through the left half of the porous membrane, mean transverse velocities of the inlet (Fig. S1c) and the vertical middle cross-section in lower channel (Fig. S1d). Flow analyses were then conducted upon these equilibrated results.

The basic features of flow field were first evaluated upon varied channel lengths with given upper and lower channel heights and membrane permeability (Fig. 2). The results showed that the transverse velocity profiles were parabolic at vertical middle cross-section of both upper and lower channels, in which the maximum value in upper channel was larger than that in lower channel (Fig. 2a). Transverse velocity along the horizontal center line exhibited that the flow flux in upper channel was gradually decreased from the inlet to the middle section and then increased to the outlet, presenting the opposite pattern with first increasing and then decreasing in lower channel (Fig. 2b). Corresponding wall shear stress of both channels showed the similar patterns with those of transverse velocity along the horizontal center line (Fig. 2c). These results showed positive correlations between central flow velocity and wall shear stress. Furthermore, the pressure difference across the porous membrane exhibited approximately linear increase from inlet to outlet (Fig. 2d). This transmembrane pressure difference resulted in that the fluid partially penetrated down into the lower channel through the left half of the porous membrane and permeated back to the upper channel on the right half (Fig. 2e), which was consistent with the flow features reported in a previous work [15] as well as the above results of flow flux, central velocity and wall shear stress. The calculating validity of transmembrane pressure difference was tested with different grid sizes from 0.5 to $4 \mu \mathrm{m}$. The results suggested the mesh independence of the calculations and the zoom-in comparisons of transmembrane pressure difference (insert) indicated that $1 \mu \mathrm{m}$ Eulerian gird size was sufficient for the calculations (Fig. 2f).

Numerical simulations upon ANSYS FLUENT were also performed for validating IBM model and MATLAB codes. The results demonstrated the similar quantifications with those of IBM simulations, especially in the case of low membrane permeability (Fig. S2). Collectively, the results indicated that the IBM model was feasible for evaluating the 
a

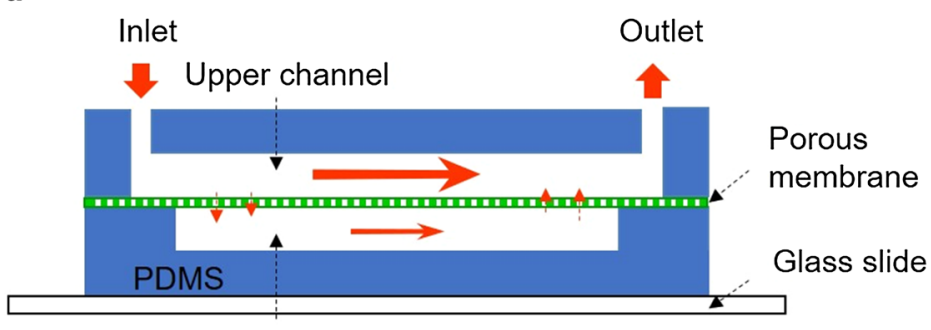

Lower channel

b

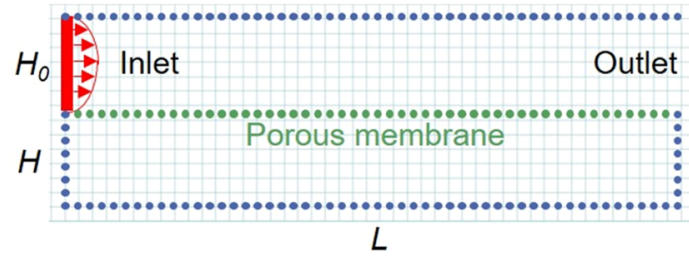

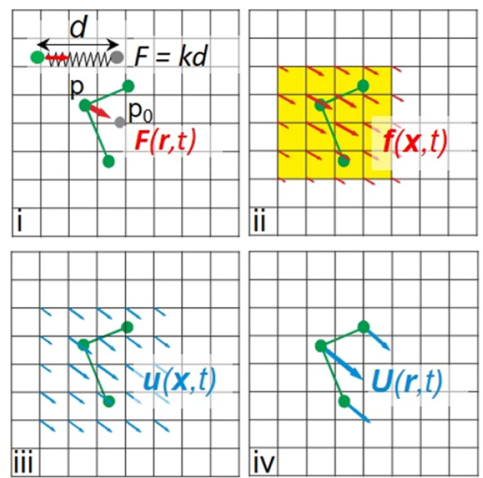

d

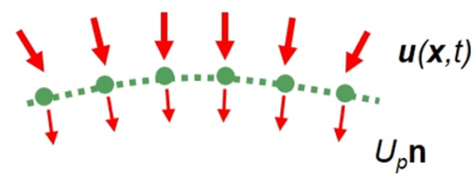

Fig. 1 Numerical modeling of a porous membrane separated, double-layered microfluidic cell co-culture chip using 2D IBM. a Schematic of experimental chip. The chip was composed of two rectangular PDMS channels separated by a porous membrane and red arrows indicated the flow directions. Fluid was forced to partially penetrate down to the lower channel through the left section of the porous membrane and flow back to the upper channel through the right section. b Simplified chip set-up for numerical modelling. Light blue meshes in background represented the computational fluid zone. Periodic boundary conditions were applied in both horizontal and vertical directions. Walls and porous membrane were both treated as immersed boundaries and represented by blue and green nodes respectively. Force was applied to the left cross-section of the upper channel for driving the flow to target speed (red arrows). $H_{0}, H$ and $L$ represented upper channel height, lower channel height and channel length, respectively. c Flowchart of numerical simulation via IBM (i-iv). For details, refer to Sect. 2.2. d Flow passing through a porous membrane with normal velocity $\boldsymbol{U}_{\mathrm{p}}$. A term of porous slip velocity $-\boldsymbol{U}_{\mathrm{p}}$ was added to the porous node to make the immersed structure permeable to fluid. $\mathbf{c}$ and d are modified from Battista's work [21]

flow features of porous membrane-separated, double-layered microfluidic chip, and the flow in both channels was governed by their transmembrane pressure difference.

\subsection{Dependence of flow features on chip geometry}

The actual magnitude of inlet transverse velocity hardly reached to the target setting and differed case by case in our IBM simulations (Fig. S3), which maybe result from the differences of flow resistance caused by different channel size and membrane permeability. Thus, the rectification was needed before comparing among different cases. The results showed that longer channels caused more deviations of inlet velocity from preset target value whereas the transmembrane pressure differences were strictly proportional to inlet mean velocity for cases of different chip geometries and membrane permeabilities (Fig. S4). In combination with the proportional relationship between the penetration velocity and transmembrane pressure difference, it could be inferred that the flow velocities of both upper and lower channels were proportional to the inlet velocity. Thus, the resulted calculations of flow flux, velocity and WSS were multiplied by a factor of $1.67 / U_{\text {in }} \mathrm{mm} / \mathrm{s}$ for comparability among the simulations with different actual inlet velocities, where $1.67 \mathrm{~mm} / \mathrm{s}$ is the mean inlet velocity corresponding to the wall shear stress of $1 \mathrm{dyn} / \mathrm{cm}^{2}\left(1 \mathrm{dyn}=1 \times 10^{-5} \mathrm{~N}\right)$ in a single-layered channel of $100 \mu \mathrm{m}$ height.

The effects of chip geometry were evaluated by varying lower channel height from 6 to $180 \mu \mathrm{m}$ and chip length from 0.8 to $13 \mathrm{~mm}$ with a constant upper channel height of $100 \mu \mathrm{m}$ and porous membrane permeability of $5 \times 10^{-13}$ $\mathrm{m}^{2}$. The results showed that, at the given chip length of $1.6 \mathrm{~mm}$, the flow flux, transverse maximum flow velocity and wall shear stress at the middle section of upper channel first decreased gradually with the increase of lower channel height and then become stable when the height was equal to or beyond that of upper channel (Fig. 3a-c, blue lines and triangles). By contrast, the flow of lower channel presented distinct features. The flow flux in lower channel first increased and became stable with the increase of lower channel height (Fig. 3a, red line and circles), presenting an opposite pattern against upper channel. Interestingly, the maximum transverse velocity and wall shear stress in lower channel exhibited obvious transition with first increase and followed decrease when the lower channel height reached a threshold (Fig. 3b, c, red lines and circles). Noting that the transverse velocity is dependent on both flow flux and channel section area and the wall shear stress relies on both centerline velocity and channel height, the transition features in lower channel was reasonable. This is because 
a

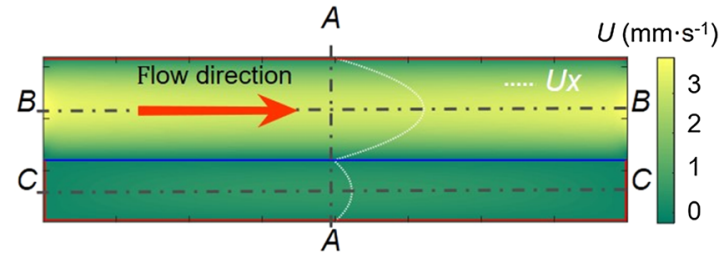

b
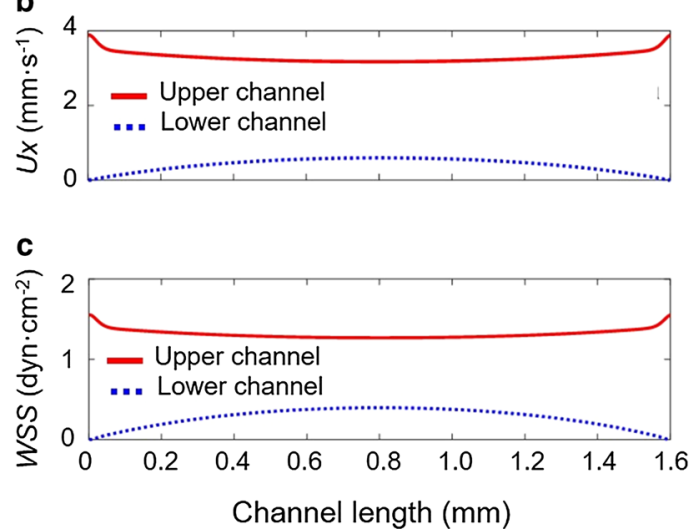

d

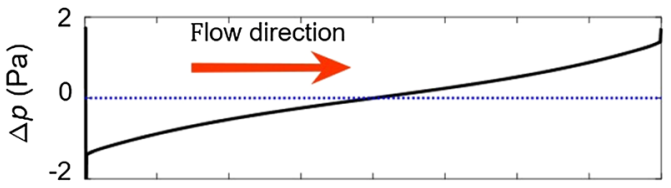

e

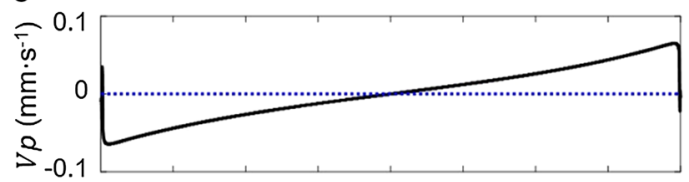

f

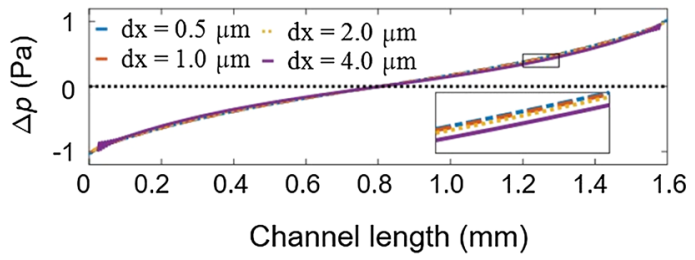

Fig. 2 Typical flow features of the microfluidic chip. a Flow profiles in the two channels (heat map) and the transverse velocity distribution at vertical middle cross-section $A$ (white curves). Channel size was not drawn in scale. b Transverse velocity distribution along the horizontal center line of upper or lower channel (dash lines of $B$ or $C$ in a). $\mathbf{c}$ Wall shear stress (WSS) distribution on the bottom surface of upper or lower channel. $\mathbf{d}$ Pressure difference and $\mathbf{e}$ penetration velocity across the membrane along the flow direction. $\mathbf{f}$ Grid independency was validated by comparing the simulations of transmembrane pressure difference at four different Eulerian mesh sizes of 0.5, 1,2 and $4 \mu \mathrm{m}$. Insert indicated the enlarged pressure drop distribution. In all calculations, parameters were set to be the upper and lower channel heights of 0.1 and $0.06 \mathrm{~mm}$, respectively, the porous membrane permeability of $5 \times 10^{-13} \mathrm{~m}^{2}$, the membrane thickness of $10 \mu \mathrm{m}$ and the fluid viscosity of $1 \mathrm{cP}$. Inlet mean velocity was $2.32 \mathrm{~mm} / \mathrm{s}$ for $\mathbf{a}-\mathbf{e}$ and $1.67 \mathrm{~mm} / \mathrm{s}$ for $\mathbf{f}$

a

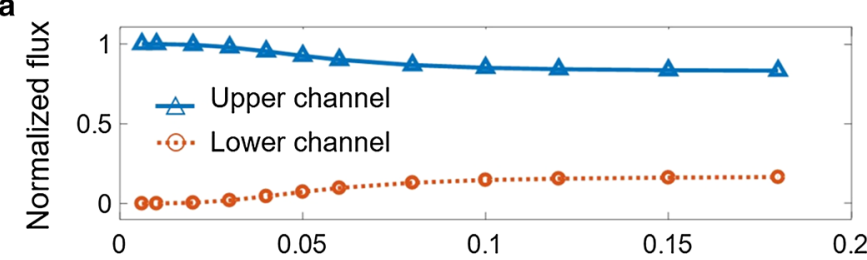

b

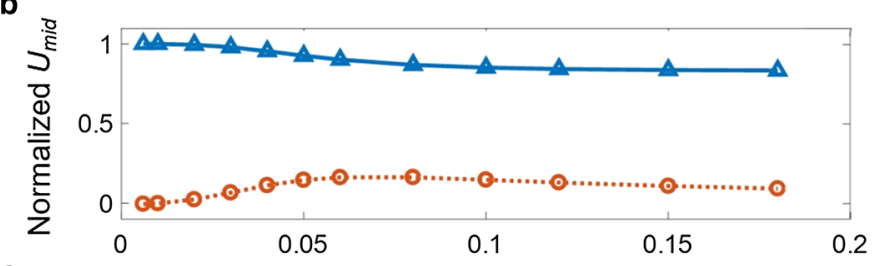

$\mathbf{C}$

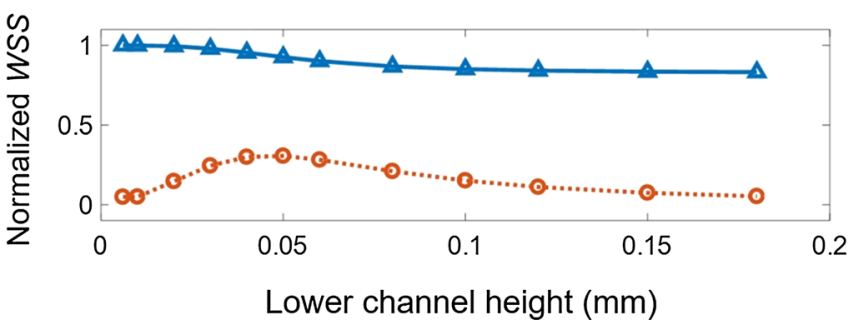

d

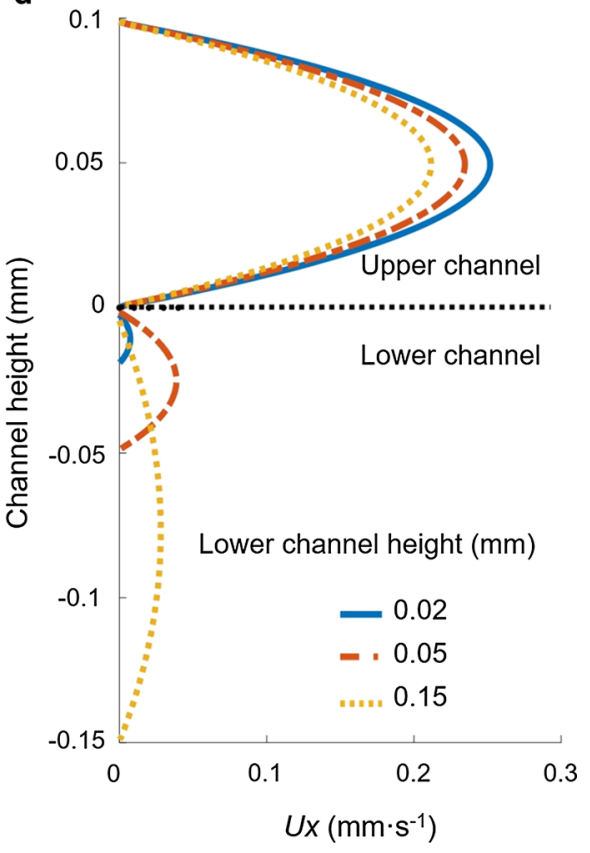

Fig. 3 Dependence of flow features on lower channel height. a-c Normalized flux (a), maximum transverse velocity $U_{\text {mid }}(\mathbf{b})$ and WSS (c) in upper (blue) or lower (red) channel at the vertical middle cross-section at different lower channel heights. For details of parameter normalization, refer to Sect. 2. d Typical transverse velocity distributions of the vertical middle cross-section at three different lower channel heights. In all calculations, parameters were set to be the upper channel height of $0.1 \mathrm{~mm}$, the channel length of $1.6 \mathrm{~mm}$, the porous membrane permeability of $5 \times 10^{-13} \mathrm{~m}^{2}$, the membrane thickness of $10 \mu \mathrm{m}$ and the fluid viscosity of $1 \mathrm{cP}$ 

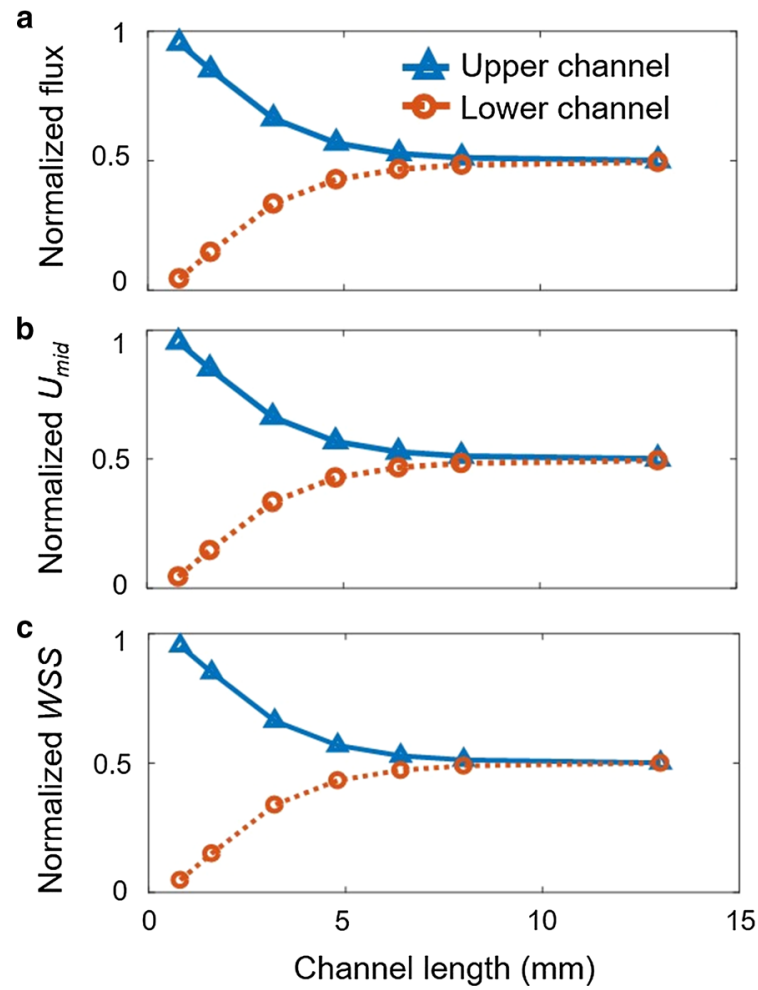

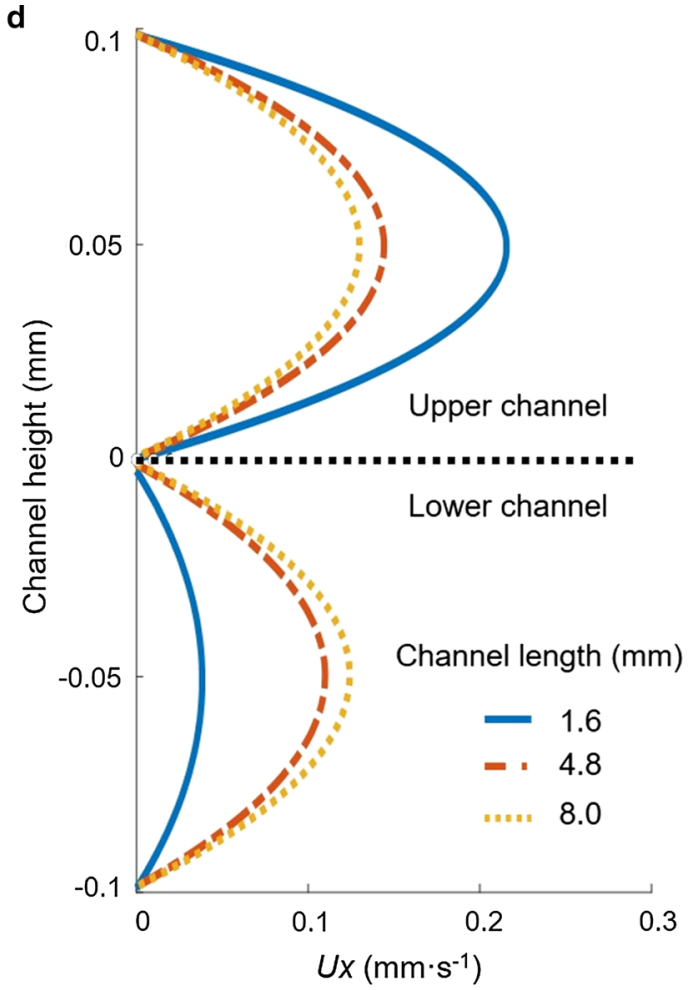

Fig. 4 Dependence of flow features on channel length. a-c Normalized flux (a), maximum transverse velocity $U_{\text {mid }}(\mathbf{b})$ and WSS (c) in upper (blue) or lower (red) channel at the vertical middle cross-section at different channel lengths. $\mathbf{d}$ Typical transverse velocity distributions of the vertical middle cross-section at three different channel lengths. In all calculations, parameters were set to be the upper and lower channels height of 0.1 and $0.1 \mathrm{~mm}$, respectively, the porous membrane permeability of $5 \times 10^{-13} \mathrm{~m}^{2}$, the membrane thickness of $10 \mu \mathrm{m}$ and the fluid viscosity of $1 \mathrm{cP}$

the increase of height would simultaneously increase flow flux and section area and the competition between the two variables determined the pattern of velocity with channel height. Similarly, the coordination between centerline velocity and channel height governed the wall shear stress. Figure $3 \mathrm{~d}$ displayed the parabolic distributions of transverse velocity at the middle section in both channels at three typical lower channel heights. It clearly showed that the peak velocity value monotonically decreased in upper channel (upper panel) and yielded a biphasic transition in lower channel (lower panel) with increase of lower channel height.

The impact of chip length on flow field was also investigated at a given lower channel height of $0.1 \mathrm{~mm}$. The results indicated that the flow flux, transverse velocity and wall shear stress at the middle section of upper channel gradually decreased with the increase of chip length and then reached a plateau when the channel was sufficiently long (Fig. 4a-c, blue lines and triangles). Those profiles in lower channel showed opposite patterns with initial increasing up to the similar plateau (Fig. 4a-c, red lines and circles). Figure $4 \mathrm{~d}$ demonstrated transverse velocity distributions at the middle section of both the channels at three typical channel lengths. Evidently, the peak velocity value presented a mono- tonic decrease in upper channel and a monotonic increase in lower channel with the increase of channel length.

Collectively, the effects of chip geometry were illustrated by combining the two variables of channel length and lower channel height (Fig. 5). With the increase of lower channel height, the wall shear stress in upper channel decreased gradually down to a plateau and the plateau value was also reduced with the increase of channel length (Fig. 5a, b). The wall shear stress in lower channel always displayed biphasic features with the increase of lower channel height and the transition point of maximum wall shear stress shifted right with the increase of channel length (Fig. $5 \mathrm{c}, \mathrm{d}$, red dash lines). In addition, different target inlet velocities did not alter the regulating patterns of chip geometry on flow features including flow flux, flow velocity and wall shear stress (Fig. S5), implying that the flow features are tightly coupled with the chip geometry.

\subsection{Dependence of flow features on membrane permeability and fluid viscosity}

The effect of porous membrane permeability was elucidated at given upper and lower channel heights of $0.1 \mathrm{~mm}$ and channel length of $8 \mathrm{~mm}$. The results showed that, with the 


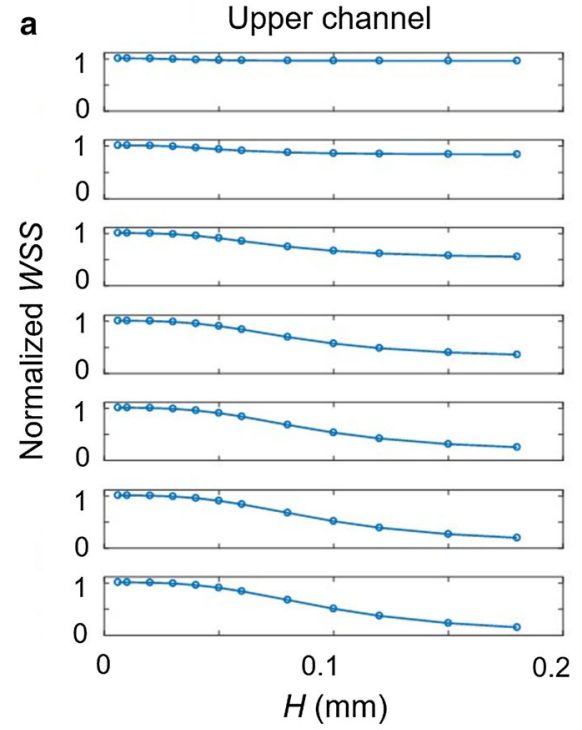

b

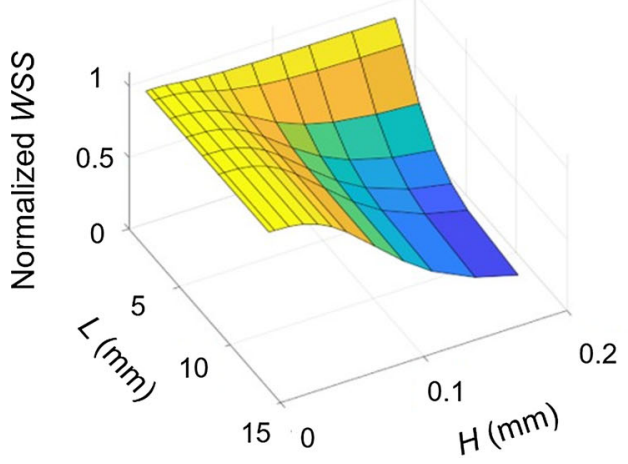

c

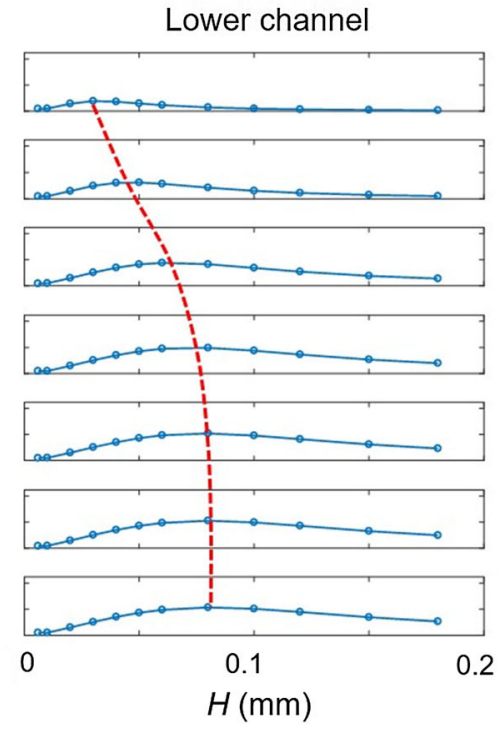

d

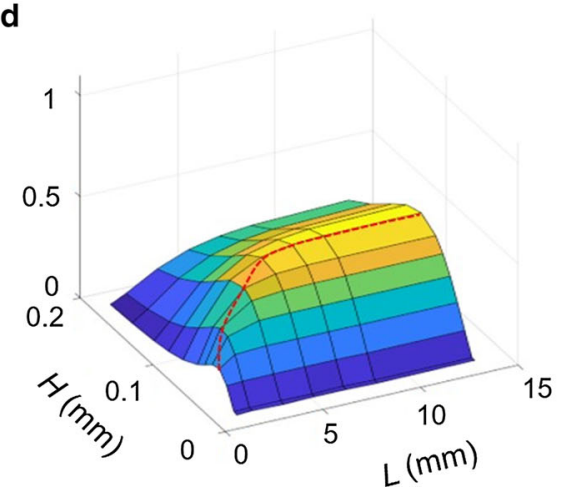

0.8

1.6

4.8

6.4

8.0

13.0

Fig. 5 Dependence of WSS on chip geometry. Impacts of both lower channel height and channel length on WSS in upper (a) or lower (c) channel were summarized and the corresponding three-dimensional profiles were shown in (b) and (d), respectively. $H$ and $L$ represented the lower channel height and the channel length respectively. Red dash lines in $\mathbf{c}$ and $\mathbf{d}$ denoted the maximum WSS with varied lower channel heights. In all calculations, parameters were set to be the upper channel height of $0.1 \mathrm{~mm}$, the porous membrane permeability of $5 \times 10^{-13} \mathrm{~m}^{2}$, the membrane thickness of $10 \mu \mathrm{m}$ and the fluid viscosity of $1 \mathrm{cP}$

increase of membrane permeability from $3 \times 10^{-16}$ to $5 \times$ $10^{-13} \mathrm{~m}^{2}$, the flow flux, transverse maximum velocity and wall shear stress at vertical middle section of upper channel decreased slightly (Fig. 6a-c, blue lines and triangles) but increased significantly in the lower channel up to the comparable values with those in upper channel (Fig. 6a-c, red lines and circles). These results were consistent with the changes in transmembrane pressure differences $(\Delta p)$, as exemplified in asterisks in Fig. 6a, and corresponding penetration velocities $\left(V_{\mathrm{p}}\right)$ with membrane permeabilities (Fig. 6d). Increasing membrane permeability evidently decreased the transmembrane pressure difference but enhanced the corresponding penetration velocity (Fig. 6d). Furthermore, reducing membrane permeability did not alter the dependence pattern of wall shear stress on chip geometry except that the transition point of maximum wall shear stress in lower channel was presented at the smaller height of lower channel, which was similar to the effect of channel length (Fig. S6). That is, for a porous membrane with lower permeability, a longer channel is needed to reach the maximum of wall shear stress in the lower channel. Thus, membrane permeability could regulate the extent but not the pattern of flow, nor the channel length does.

Viscosity of blood varies largely in many pathophysiological processes such as nonalcoholic fatty liver disease [23]. Thus, we further investigated the influence of fluid viscosity on flow features. The results displayed that the transmembrane pressure difference and wall shear stress increased with the increase of fluid viscosity (Fig. 7a, b) but the vertical transmembrane velocity and horizontal flow flux remained the same (Fig. 7c, d). This is reasonable because the transmembrane pressure difference is determined by the fluid viscosity at a constant inlet flow flux and the wall shear stress is proportional to fluid viscosity at the same shear rate. 


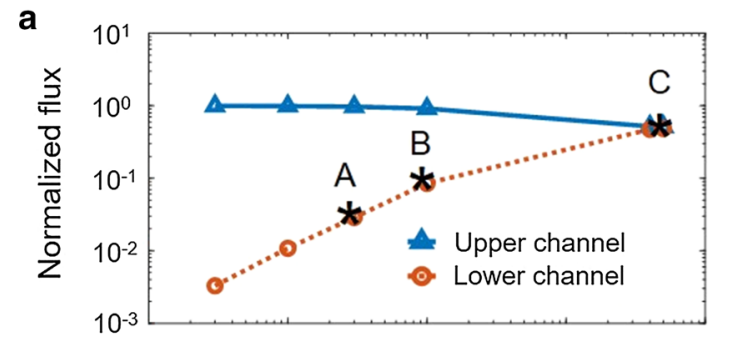

d
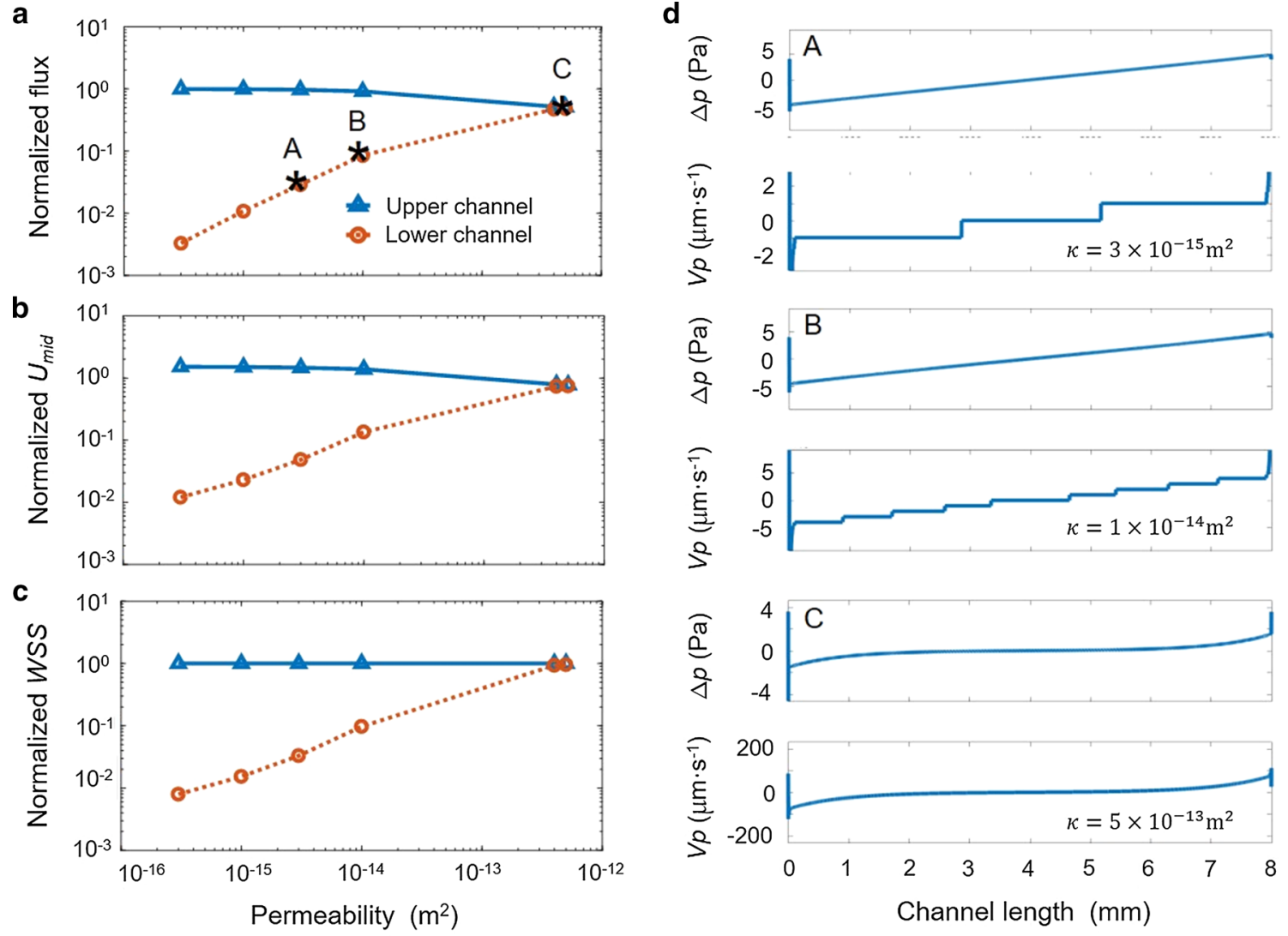

Fig. 6 Dependence of flow features on porous membrane permeability. a-c Normalized flux (a), maximum transverse velocity $U_{\text {mid }}(\mathbf{b})$ and WSS (c) at the vertical middle cross-section in upper (blue) and lower (red) channel against porous membrane permeability. d Distributions of transmembrane pressure difference $\Delta p$ and corresponding flow penetration velocity $V_{\mathrm{p}}$ across the membrane at three typical permeabilities (black asterisks in a) of $3 \times 10^{-15}(A), 1 \times 10^{-14}(B)$ and $5 \times 10^{-13} \mathrm{~m}^{2}(C)$. The heights of upper and lower channel were both set as $0.1 \mathrm{~mm}$, and the membrane thickness and fluid viscosity were $10 \mu \mathrm{m}$ and $1 \mathrm{cP}$, respectively

Accordingly, it could be inferred that the hepatocytes may suffer the increase of wall shear stress in the liver upon the increase of blood viscosity and the unchanged permeability of the sinusoidal endothelium.

\subsection{Analytical model of an analogical porous membrane-separated, double-layered microfluidic chip}

To quantitatively describe the relationships among flow features, chip geometry and porous membrane permeability, a 2D analytical model was further developed in this study to predict the distribution of transmembrane pressure difference along the flow direction and deduce the distributions of wall shear stress in both upper and lower channels. A brief formulation of the analytical model was outlined below and the corresponding derivations were elaborated in Supplemental document 1 .

The development of analytical model was based on two prerequisites. The first one was that the transverse velocity distributions of the flow in either upper or lower channel were approximated as a Poiseuille flow, which had been proved by the above IBM simulations (Fig. 2a). The other was that the channel width was much larger than channel heights (Fig. 8a) and the flow along channel width was constant. Thus, the transverse velocity $u$ and flow flux $q$ in upper and lower channels were respectively written as follows,

$u_{1}=-\frac{1}{4 \mu} \frac{\mathrm{d} p_{1}}{\mathrm{~d} x} y\left(H_{1}-y\right)$,

$q_{1}=\int_{0}^{H_{1}} u_{1} \cdot b \mathrm{~d} y=-\frac{b H_{1}^{3}}{24 \mu} \frac{\mathrm{d} p_{1}}{\mathrm{~d} x}$,

$u_{2}=\frac{1}{4 \mu} \frac{\mathrm{d} p_{2}}{\mathrm{~d} x} y\left(H_{2}+y\right)$,

$q_{2}=\int_{0}^{H_{2}} u_{2} \cdot b \mathrm{~d} y=-\frac{b H_{2}^{3}}{24 \mu} \frac{\mathrm{d} p_{2}}{\mathrm{~d} x}$,

where $b$ was the channel width, and subscripts "1" and "2" represent the upper and lower channels, respectively. Set the 

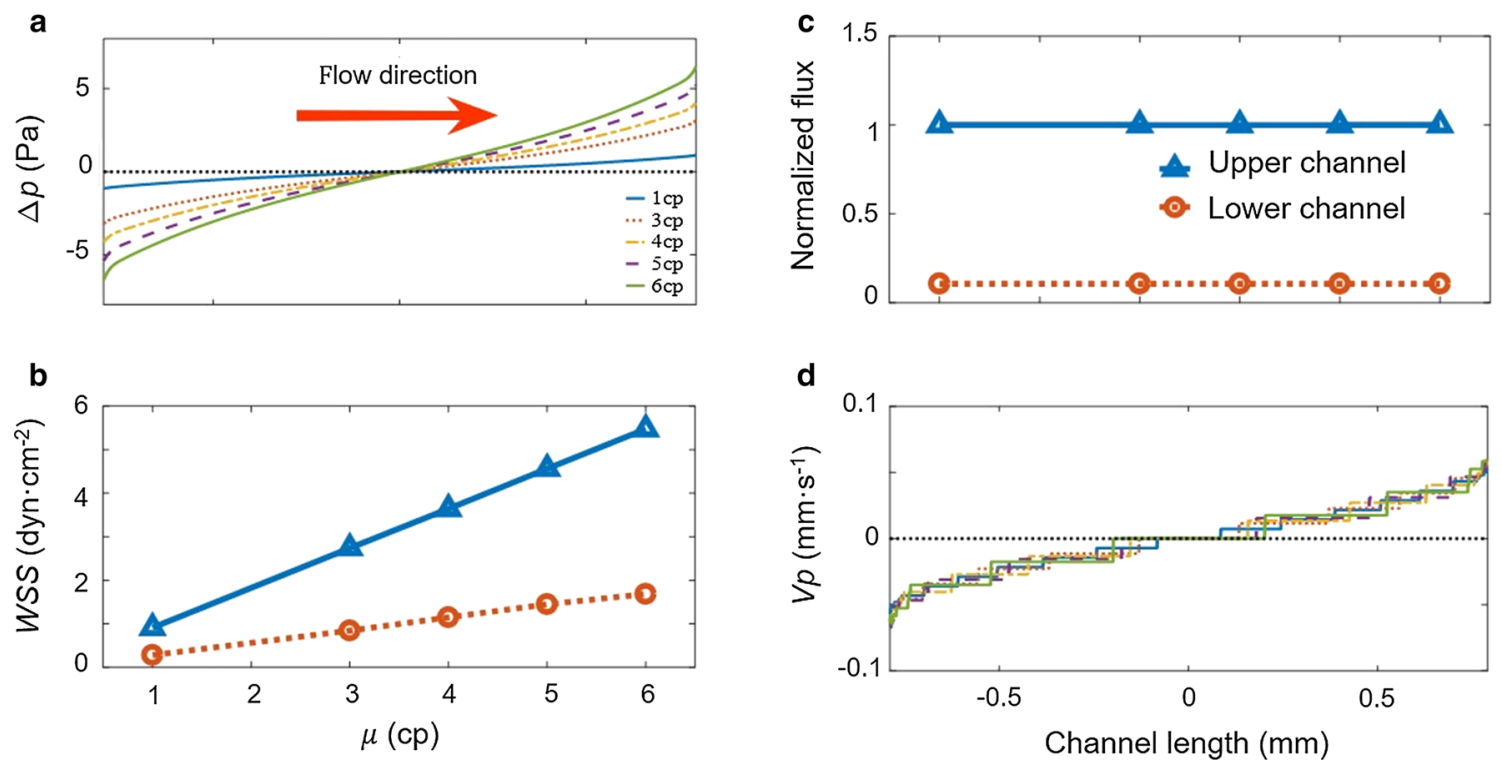

Fig. 7 Effect of fluid viscosity on flow features. a Distribution of transmembrane pressure difference along the flow direction at different fluid viscosities. b, c Dependences of WSS (b) and normalized flow flux (c) on different fluid viscosities. d Distribution of penetration velocity difference along the flow direction at different fluid viscosities. The upper, lower channel heights and channel length were set as $0.1,0.06$ and $1.6 \mathrm{~mm}$, respectively, and the porous membrane permeability was $5 \times 10^{-13} \mathrm{~m}^{2}$

inlet flow flux as $q_{0}$, which could be written upon the flux conservation condition as:

$q_{0}=q_{1}+q_{2}$.

The transmembrane pressure difference was defined as $p_{\perp}(x)=p_{2}-p_{1}$ and proportional to the transmembrane flow velocity $V_{\mathrm{p}}$ according to Darcy's law,

$-\frac{\mathrm{d} q_{2}}{b \mathrm{~d} x}=V_{\mathrm{p}}(x)=p_{\perp}(x) \cdot \lambda$

where $\lambda$ was the porous slip parameter, which could be estimated from the membrane permeability $\kappa$ and membrane thickness $h$ by $\lambda=\kappa /(\mu h)$. Based on above equations, a second-order homogeneous linear differential equations with constant coefficients was derived,

$\frac{\mathrm{d}^{2} p_{\perp}}{\mathrm{d} x^{2}}-a p_{\perp}=0$,

where

$a=24 \mu \lambda \cdot \frac{H_{1}^{3}+H_{2}^{3}}{H_{1}^{3} H_{2}^{3}}=\frac{24 \kappa}{h} \cdot \frac{H_{1}^{3}+H_{2}^{3}}{H_{1}^{3} H_{2}^{3}}$.

Considering two boundary conditions at the inlet and the middle of channel,

$\left.p_{\perp}\right|_{x=0}=p_{\perp 0}$,

$\left.p_{\perp}\right|_{x=L / 2}=0$, the solution for the above equation was derived as

$p_{\perp}(x)=\frac{p_{\perp 0}}{1-\mathrm{e}^{\sqrt{a} L}}\left[\mathrm{e}^{\sqrt{a} x}-\mathrm{e}^{\sqrt{a}(L-x)}\right]$.

Equation (17) denoted the transmembrane pressure difference distribution as a function of the inlet transmembrane pressure difference $p_{\perp 0}$, the membrane permeability $\kappa$ (included in $a$ ) and channel geometry $\left(H_{1}, H_{2}\right.$ and $\left.L\right)$. To determine the inlet transmembrane pressure difference $p_{\perp 0}$, the flow flux of both channels was first derived as:

$q_{1}=q_{0}-\frac{b \lambda p_{\perp 0}}{\sqrt{a}\left(1-\mathrm{e}^{\sqrt{a} L}\right)}\left[1+\mathrm{e}^{\sqrt{a} L}-\mathrm{e}^{\sqrt{a} x}-\mathrm{e}^{\sqrt{a}(L-x)}\right]$,

$q_{2}=\frac{b \lambda p_{\perp 0}}{\sqrt{a}\left(1-\mathrm{e}^{\sqrt{a} L}\right)}\left[1+\mathrm{e}^{\sqrt{a} L}-\mathrm{e}^{\sqrt{a} x}-\mathrm{e}^{\sqrt{a}(L-x)}\right]$.

And the inlet transmembrane pressure difference was:

$p_{\perp 0}=\frac{24 \mu q_{0}}{b H_{1}^{3}} \cdot \frac{1-\mathrm{e}^{\sqrt{a} L}}{\sqrt{a}\left(1+\mathrm{e}^{\sqrt{a} L}\right)}$.

In conclusion, the transmembrane pressure difference along the flow direction was:

$p(x)=\frac{24 \mu q_{0}}{b H_{1}^{3}} \cdot \frac{\mathrm{e}^{\sqrt{a} x}-\mathrm{e}^{\sqrt{a}(L-x)}}{\sqrt{a}\left(1+\mathrm{e}^{\sqrt{a} L}\right)}$. 
a
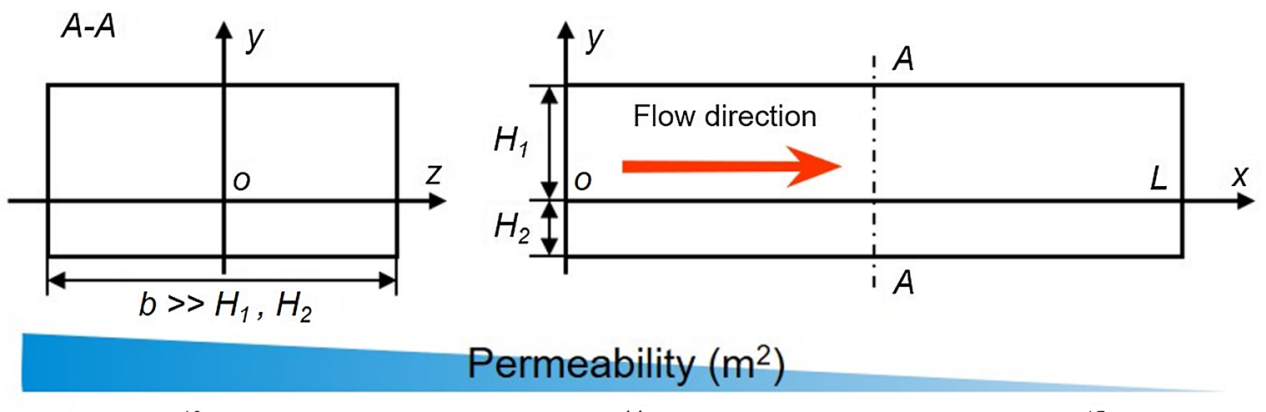

$5 \times 10^{-13}$

$1 \times 10^{-14}$

$1 \times 10^{-15}$
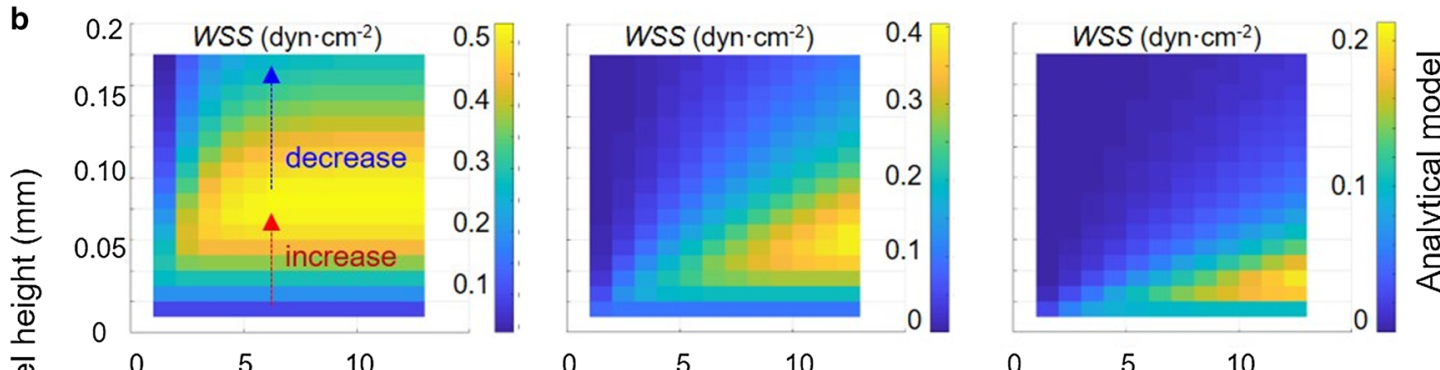

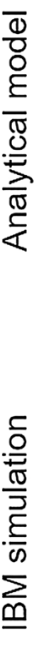

0
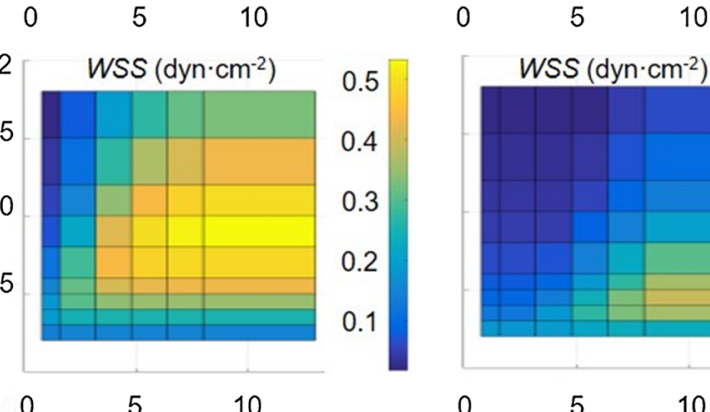

$5 \quad 10$
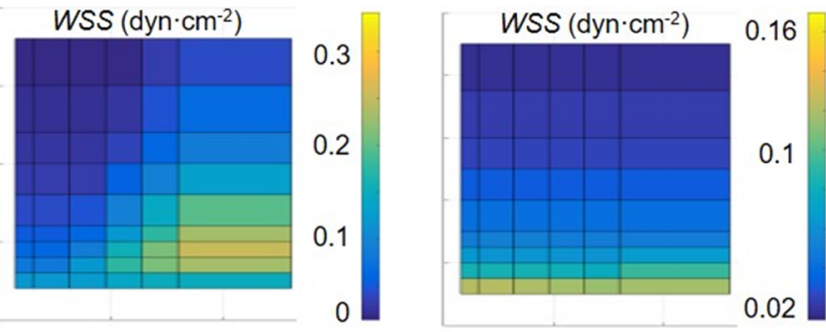

Channel length $(\mathrm{mm})$
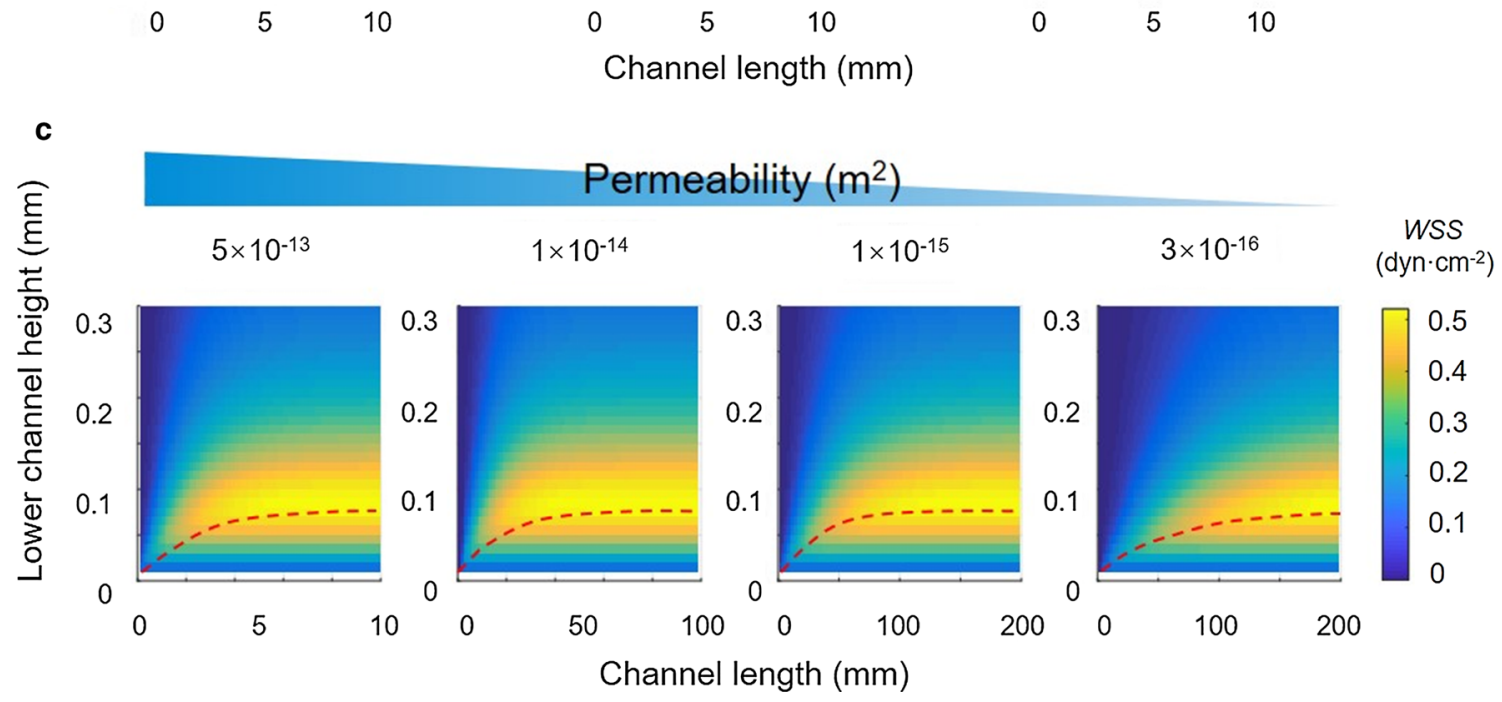

Fig. 8 Analytical model of an analogical porous membrane separated, double-layer microfluidic cell co-culture chip. a Schematic and geometry of the chip. b Comparisons of WSS between IBM simulation and analytical solution. Arrows in upper left depicted their biphasic distributions. c Theoretical predictions for the dependence of lower channel WSS on chip geometry and membrane permeability. Red dash lines indicated the WSS peak values. Mean value of inlet velocity was set to $1.67 \mathrm{~mm} / \mathrm{s}$ 
The wall shear stress in both upper and lower channel could be written as

$$
\begin{aligned}
\tau_{1} & =\frac{6 \mu q_{0}}{b H_{1}^{2}}\left[\frac{H_{1}^{3}}{H_{1}^{3}+H_{2}^{3}}+\frac{H_{2}^{3}}{H_{1}^{3}+H_{2}^{3}} \frac{\mathrm{e}^{\sqrt{a}(L-x)}+\mathrm{e}^{\sqrt{a} x}}{\mathrm{e}^{\sqrt{a} L}+1}\right], \\
\tau_{2} & =\frac{-6 \mu q_{0}}{b} \cdot \frac{H_{2}}{H_{1}^{3}+H_{2}^{3}} \cdot\left[\frac{\mathrm{e}^{\sqrt{a} x}+\mathrm{e}^{\sqrt{a}(L-x)}}{\left.1+\mathrm{e}^{\sqrt{a} L}-1\right] .},\right.
\end{aligned}
$$

Based on Eqs. (22, 23), it was evident that the wall shear stresses of both upper and lower channels were tightly coupled with chip geometry and membrane permeability. Theoretical solutions presented the consistent patterns with those of IBM simulations, indicating that the increase of lower channel height resulted in a biphasic pattern of wall shear stress distribution with first increase and followed decrease. They also demonstrated that the increases of channel length and membrane permeability enlarged the transition threshold of maximum shear stress but not the biphasic pattern (Fig. 8b). The maximum shear stress in lower channel was presented around the same lower channel height and was independent of membrane permeability when the chip was sufficiently long (Fig. 8c). In combination of IBM simulations and theoretical analyses, the results further indicated that flow features of an analogical porous membrane-separated, double-layered microfluidic chip were sensitive to chip geometry and membrane permeability.

\section{Discussion}

Porous membrane-separated, double-layered microfluidic systems are widely used for tissue barrier study and cell coculture models [1-3, 16-20, 24-26]. However, their fluid characteristics have not been systematically studied because of the lack of criteria for membrane porosity selection and channel size design. This work investigated the flow feature dependence on chip geometry and membrane permeability upon IBM simulations. The results exhibited the monotonic pattern of wall shear stress in upper channel with varied lower channel height, channel length as well as membrane permeability, and the biphasic pattern of wall shear stress in lower channel. A theoretical model was also developed for quantitatively describing the relationship between flow features and chip properties. This work clarified the regulation of channel length and height and membrane permeability on flow flux and wall shear stresses of the channels, providing the bases for evaluating flow features of similar chips upon chip geometry and membrane permeability and promoting the flow-specified chip design.

Roles of shear flow in biological functions are widely studied in organ development [9], tissue homeostasis [10, 12] and cell regulation $[11,12]$. While the effects of chip geometry and membrane permeability on flow features are still unclear, existing evidences provide certain cues in their correlations. For example, a $24 \mu \mathrm{m}$-thick membrane with a porosity of $0.44 \%$ and a pore diameter of $0.75 \mu \mathrm{m}$ was used in a doublelayer chip to achieve a rapid but shear-free delivery of solutes to the cells cultured in lower channel [27]. This design was improved to reduce transmembrane diffusion time from $\sim 60 \mathrm{~s}$ to $10 \mu \mathrm{s}$, using an ultrathin membrane of $15 \mathrm{~nm}$-thickness, $30 \mathrm{~nm}$-pore size and $10 \%$ porosity laid on a $120 \mathrm{~nm}$ thick SiN scaffold, and keep the same hydraulic resistance level [15]. Furthermore, flow features of a porous membrane separated, double-layered channel were analyzed by an electrical circuit analogy model, in which the corresponding COMSOL simulations and experimental results validated the dramatic reduction of shear flow in the cell culture chamber with sufficiently short channel and low membrane porosity [12, 24]. Those studies also showed that the flow flux in lower channel reached a plateau value that was independent of membrane permeability when the channel length is sufficiently long. And setting the channel length much shorter than that needed for reaching plateau was key for getting a shear-free lower channel [15, 25]. However, the impacts of channel height, as well as the correlation among channel height, length and membrane permeability on flow features are still unknown.

In this study, we systematically investigated the impacts of the three major factors on channel flows and focused on their roles in regulating wall shear stresses in both upper and lower channels. Our simulations clearly elucidated the dependences of flow flux, wall shear stress, and transmembrane pressure difference on chip geometry (channel height and length) and membrane permeability. The results further indicated that sufficient channel length required for achieving a stable plateau in lower channel was tightly coupled with membrane permeability and channel height. For a lower permeability membrane, a longer channel was favorable to achieve a permeability-independent plateau. An interesting result was that the enhancement of lower channel height led to first increase then decrease of wall shear stress in a biphasic pattern and the threshold of maximum wall shear stress was dependent on both channel length and membrane permeability. Analytical solutions on transmembrane pressure difference and wall shear stress distributions also provided the references to evaluate the flow features of existing chips or to optimize the design of a porous membrane-separated, double-layered microfluidic chip. While IBM method is normally used for investigating elastic structure-fluid interaction, only chip fluid features were focused here but not the deformation of the elastic porous membrane. Thus the membrane was simplified as a series of constrained points using stiff springs with their displacements not beyond one Eulerian mesh grid, and the mesh grid was set small enough for the calculation stability and convergence. This simplification 
was reasonable because the elasticity of the porous membrane must be stiff enough in chip for avoiding collapse with cell seeding or chip lengthening. Furthermore, the heights of upper and lower channels of the two-layer chip were far larger than the membrane thickness, which also weakened the effect of membrane deformation on fluid features. The rationality of membrane simplification in IBM simulations was also validated upon the comparable results with those of Fluent simulations, in which the latter treated the porous membrane just as inelastic porous zone (Fig. S2). Overall, our IBM numerical simulations agreed well with the corresponding analytical models and previous numerical studies. In addition, the IBM simulations in this study were consistent with the experimental particle tracking visualization (PTV) results (Fig. S7) [1].

However, a few unexpected phenomena were also observed due to the limitations of numerical simulation method used here. Firstly, there existed slight reverse flows near the immersed boundaries, presumably resulting from approximation of no-slip boundary condition that is realized by pre-calculated restoring force [28]. Although several methods have been introduced for overcoming this deficiency $[29,30]$, these improved methods were not applied in this study with the consideration of reducing computational complexity. In addition, this limitation did not alter the patterns of calculated flow features even with moderate underestimation of lower channel flow. Secondly, the penetration velocity distributions were not always smooth along the porous membrane in a stepwise manner at low membrane permeabilities, not like the stable distributions of transmembrane pressure difference (Fig. S8). This inaccuracy may affect the exact values, but not the patterns, of fluid flow. In addition, our analytical model could serve as a good complement to this deficiency of IBM simulation.

Collectively, treating the porous membrane as an immersed boundary was effective in studying the flow features of a porous membrane-separated, double-layered microfluidic chip. This study proved the sensitivity of chip geometry and membrane permeability on flow features, established the quantitative formulations for describing the correlations between flow fields and chip features, and offered the criteria for optimizing the flow-specified chip design. It can be easily expanded to evaluate the flow features of physiological or pathological liver sinusoids or other tissues in vivo.

Acknowledgements We thank Drs. Beiji Shi and Shizhao Wang for helpful discussions. The simulations were performed on the HighPerformance Computing Center of Collaborative Innovation Center of Advanced Microstructures in Nanjing. This work was supported by the National Natural Science Foundation of China (Grants 91642203, 31627804, 31661143044, and 31570942), the Frontier Science Key Project of Chinese Science Academy (Grant QYZDJ-SSW-JSC018), and the Strategic Priority Research Program of Chinese Academy of Sciences (Grant XDB22040101).

\section{References}

1. Du, Y., Li, N., Yang, H., et al.: Mimicking liver sinusoidal structures and functions using a 3D-configured microfluidic chip. Lab Chip 17, 782-794 (2017). https://doi.org/10.1039/c6lc01374k

2. Jang, K.J., Suh, K.Y.: A multi-layer microfluidic device for efficient culture and analysis of renal tubular cells. Lab Chip 10, 36-42 (2010). https://doi.org/10.1039/b907515a

3. Huh, D., Matthews, B.D., Mammoto, A., et al.: Reconstituting organ-level lung functions on a chip. Science 328, 1662-1668 (2010). https://doi.org/10.1126/science. 1188302

4. Sung, J.H., Yu, J.J., Luo, D., et al.: Microscale 3-D hydrogel scaffold for biomimetic gastrointestinal (GI) tract model. Lab Chip 11, 389-392 (2011). https://doi.org/10.1039/c01c00273a

5. Huh, D., Torisawa, Y.S., Hamilton, G.A., et al.: Microengineered physiological biomimicry: organs-on-chips. Lab Chip 12, 2156-2164 (2012). https://doi.org/10.1039/c2lc40089h

6. Guguen-Guillouzo, C., Clement, B., Baffet, G., et al.: Maintenance and reversibility of active albumin secretion by adult rat hepatocytes co-cultured with another liver epithelial cell type. Exp. Cell Res. 143, 47-54 (1983). https://doi.org/10.1016/0014-4827(83)90 107-6

7. Yeon, J.H., Park, J.K.: Microfluidic cell culture systems for cellular analysis. Biochip J. 1, 17-27 (2007)

8. Kim, S.S., Utsunomiya, H., Koski, J.A., et al.: Survival and function of hepatocytes on a novel three-dimensional synthetic biodegradable polymer scaffold with an intrinsic network of channels. Ann. Surg. 228, 8-13 (1998). https://doi.org/10.1097/00000658-19980 7000-00002

9. Lorenz, L., Axnick, J., Buschmann, T., et al.: Mechanosensing by beta1 integrin induces angiocrine signals for liver growth and survival. Nature 562, 128-132 (2018). https://doi.org/10.1038/s4158 6-018-0522-3

10. Zhong, M., Komarova, Y., Rehman, J., et al.: Mechanosensing Piezo channels in tissue homeostasis including their role in lungs. Pulm. Circ. 8, 6 (2018). https://doi.org/10.1177/20458940187673 93

11. Kawata, K., Aoki, S., Futamata, M., et al.: Mesenchymal cells and fluid flow stimulation synergistically regulate the kinetics of corneal epithelial cells at the air-liquid interface. Graefes Arch. Clin. Exp. Ophthalmol. 257, 1915-1924 (2019). https://doi.org/1 0.1007/s00417-019-04422-y

12. Humphrey, J.D.: Vascular adaptation and mechanical homeostasis at tissue, cellular, and sub-cellular levels. Cell Biochem. Biophys. 50, 53-78 (2008). https://doi.org/10.1007/s12013-007-9002-3

13. Ong, L.J.Y., Ching, T., Chong, L.H., et al.: Self-aligning Tetris-Like (TILE) modular microfluidic platform for mimicking multi-organ interactions. Lab Chip 19, 2178-2191 (2019). https://doi.org/10.1 039/c91c00160c

14. Santhanam, N., Kumanchik, L., Guo, X.F., et al.: Stem cell derived phenotypic human neuromuscular junction model for dose response evaluation of therapeutics. Biomaterials 166, 64-78 (2018). https://doi.org/10.1016/j.biomaterials.2018.02.047

15. Chung, H.H., Mireles, M., Kwarta, B.J., et al.: Use of porous membranes in tissue barrier and co-culture models. Lab Chip 18, 1671-1689 (2018). https://doi.org/10.1039/c7lc01248a

16. Carter, R.N., Casillo, S.M., Mazzocchi, A.R., et al.: Ultrathin transparent membranes for cellular barrier and co-culture models. Biofabrication 9, 13 (2017). https://doi.org/10.1088/1758-509 0/aa5ba7 
17. Huh, D., Leslie, D.C., Matthews, B.D., et al.: A human disease model of drug toxicity-induced pulmonary edema in a lung-on-achip microdevice. Sci. Transl. Med. 4, 8 (2012). https://doi.org/10. 1126/scitranslmed.3004249

18. Kang, Y.B., Sodunke, T.R., Lamontagne, J., et al.: Liver sinusoid on a chip: long-term layered co-culture of primary rat hepatocytes and endothelial cells in microfluidic platforms. Biotechnol. Bioeng. 112, 2571-2582 (2015). https://doi.org/10.1002/bit.25659

19. Prodanov, L., Jindal, R., Bale, S.S., et al.: Long-term maintenance of a microfluidic 3D human liver sinusoid. Biotechnol. Bioeng. 113, 241-246 (2016). https://doi.org/10.1002/bit.25700

20. Deng, J., Zhang, X.L., Chen, Z.Z., et al.: A cell lines derived microfluidic liver model for investigation of hepatotoxicity induced by drug-drug interaction. Biomicrofluidics (2019). https://doi.org/ 10.1063/1.5070088

21. Battista, N.A., Strickland, W.C., Miller, L.A.: IB2d: a Python and MATLAB implementation of the immersed boundary method. Bioinspir. Biomim. (2017). https://doi.org/10.1088/1748-3190/aa5 e08

22. Kim, Y., Peskin, C.S.: 2-D parachute simulation by the immersed boundary method. SIAM J. Sci. Comput. 28, 2294-2312 (2006). https://doi.org/10.1137/S1064827501389060

23. Zhao, H.Y., Li, J., Xu, M., et al.: Elevated whole blood viscosity is associated with insulin resistance and non-alcoholic fatty liver. Clin. Endocrinol. 83, 806-811 (2015). https://doi.org/10.1111/cen. 12776

24. Ma, S.H., Lepak, L.A., Hussain, R.J., et al.: An endothelial and astrocyte co-culture model of the blood-brain barrier utilizing an ultra-thin, nanofabricated silicon nitride membrane. Lab Chip 5, 74-85 (2005). https://doi.org/10.1039/b405713a
25. Chung, H.H., Chan, C.K., Khire, T.S., et al.: Highly permeable silicon membranes for shear free chemotaxis and rapid cell labeling. Lab Chip 14, 2456-2468 (2014). https://doi.org/10.1039/c4lc003 26h

26. Bhatia, S.N., Ingber, D.E.: Microfluidic organs-on-chips. Nat. Biotechnol. 32, 760-772 (2014). https://doi.org/10.1038/nbt.298 9

27. VanDersarl, J.J., Xu, A.M., Melosh, N.A.: Rapid spatial and temporal controlled signal delivery over large cell culture areas. Lab Chip 11, 3057-3063 (2011). https://doi.org/10.1039/c1lc20311h

28. Shu, C., Liu, N., Chew, Y.T.: A novel immersed boundary velocity correction-lattice Boltzmann method and its application to simulate flow past a circular cylinder. J. Comput. Phys. 226, 1607-1622 (2007). https://doi.org/10.1016/j.jcp.2007.06.002

29. Kim, J., Kim, D., Choi, H.: An immersed-boundary finite-volume method for simulations of flow in complex geometries. J. Comput. Phys. 171, 132-150 (2001). https://doi.org/10.1006/jcph.2001.67 78

30. Wang, X., Shu, C., Wu, J., et al.: A boundary conditionimplemented immersed boundary-lattice boltzmann method and its application for simulation of flows around a circular cylinder. Adv. Appl. Math. Mech. 6, 811-829 (2015). https://doi.org/10.42 08/aamm.2013.m-s2 